\title{
Response to Letter to Editor
}

Sir,

We thank Dr. Hadigal for his interest in our work and for picking up a typographical error in Table 3.

With regard to his comment on duration of therapy for GT-1 and 4 HCV infection in ribavirin (RBV)-intolerant patients with decompensated liver cirrhosis, we would like to recapitulate that

1. The length of treatment (12 vs. 24 weeks) and the need for RBV in patients with GT-1 and 4 infection were evaluated in the ION-1 trial which reported SVR12 rates of $97-99 \%$ across all arms, with no difference in SVR based on length of treatment, use of ribavirin, or HCV genotype 1 subtype. Sixteen percent of the patients were cirrhotic. There was no difference in SVR12 rate in those with cirrhosis (97\%) vs. those without cirrhosis (98\%). Thus, 12 weeks' duration of therapy with $\mathrm{SOF}+\mathrm{LDV}$, with or without RBV is adequate for CHC and compensated LC due to GT-1 or 4 infection.

2. However, in decompensated cirrhosis, the benefit of RBV addition has been shown by the SOLAR-1 and SOLAR- 2 trials, the NHS expanded access (UK) as well as the French early access programs. Studies have shown that extending therapy to 24 weeks and use of RBV improved SVR.

3. As a general principle, regimen containing SOF $+\mathrm{LDV}$ or DCV along with ribavirin are recommended for 12 weeks and regimen containing SOF + LDV or DCV without ribavirin are recommended for 24 weeks. This has been followed in Table 2, for genotype 1 patients with cirrhosis, which mentions that the recommended regimen are SOF + LDV or DCV with RBV for 12 weeks or SOF + LDV or DCV with RBV $\times 24$ weeks. The text has highlighted the increased efficacy of RBV containing regimen. However in Table 3 about alternative regimens for RBV-intolerant patients with decompensated cirrhosis, there is a typographic error and SOF + LDV without RBV should be for 24 weeks rather than 12 weeks.

4. With reference to the anti-HCV test being negative by ELISA in patients with HIV, the point made is valid but is already covered in the manuscript. Page 120 clearly mentions that in patients with HIV, HCVRNA may be required for diagnosis if anti-HCV is negative.

Sincerely,

Panakj Puri, Vivek Saraswat For INASL HCV Guidance Group

E-mail: profviveksaraswat@gmail.com (V. Saraswat)

http://dx.doi.org/10.1016/j.jceh.2016.09.008 